\title{
Acetolysis of waste PET for upcycling and life-cycle assessment study
}

\author{
Yuantao Peng \\ University of Science and Technology of China \\ Jie Yang \\ University of Science and Technology of China \\ Chenqiang Deng \\ University of Science and Technology of China \\ Yao Fu \\ University of Science and Technology of China \\ Jin Deng ( $\nabla$ dengjin@ustc.edu.cn )
}

University of Science and Technology of China https://orcid.org/0000-0003-0272-1031

Article

Keywords:

Posted Date: February 7th, 2022

DOI: https://doi.org/10.21203/rs.3.rs-1275958/v1

License: (c) (1) This work is licensed under a Creative Commons Attribution 4.0 International License.

Read Full License 


\section{Acetolysis of waste PET for upcycling and life-cycle assessment study}

${ }^{1}$ Y. Peng, J. Yang, C. Deng, Yao Fu and J. Deng

4 CAS Key Laboratory of Urban Pollutant Conversion, Anhui Province Key Laboratory of Biomass Clean Energy,

5 Department of Applied Chemistry, University of Science and Technology of China, Hefei, Anhui, 230026, PR China

$6 \quad$ Yuantao Peng, E-mail: pengyt023@ mail.ustc.edu.cn

7 Jie Yang, E-mail: yangjieustc@ mail.ustc.edu.cn

8 Chenqiang Deng, E-mail: dengchqi@mail.ustc.edu.cn

$9 \quad *$ Corresponding author

10 E-mail: fuyao@ustc.edu.cn

11 E-mail: dengjin@ustc.edu.cn

12 Abstract

To reduce environmental pollution and reliance on fossil resources, polyethylene terephthalate (PET) as the most consumed synthetic polyester needs to be recycled effectively. However, the existing recycling methods cannot process colored or blended PET materials for upcycling. Here we report a new efficient method for acetolysis of waste PET into terephthalic acid (TPA) and ethylene glycol diacetate (EGDA) in acetic acid. Since acetic acid can dissolve or decompose other components such as dyes, additives, blends, etc., TPA can be crystallized out in a high-purity form. On the other hand, EGDA can be hydrolyzed to ethylene glycol or directly polymerized with TPA to form PET, completing the closed-loop recycling. The life-cycle assessment shows that, compared with the petroleum route, $67 \%$ of global warming potential and

$2077 \%$ of non-renewable energy use can be reduced via acetolysis under optimum conditions. This is the lowest

21 environmental impact of all chemical recycling options available today. 
Plastics have become an important material in the modern global consumer economy, and the production is estimated to increase to around 12 billion tons by $2050^{1-4}$. As a large class of plastics, polyethylene terephthalate (PET) is widely used in various containers, packaging materials, and textiles ${ }^{5-7}$, with a production scale of more than 70 million tons per year ${ }^{8}$. Due to the material characteristics of PET, it is difficult to degrade in nature and has a low recycling rate, resulting in a large amount of plastic waste ${ }^{9-11}$. Therefore, effective recycling or upcycling methods for waste plastic are being intensively developed ${ }^{12-14}$.

At present, post-consumer PET is mainly recycled by mechanical methods, but this will lead to deterioration of material structure and performance, and can only produce low-value products for downcycling ${ }^{6,15}$. Compared with mechanical recycling, PET can be converted into monomers for polymerization or other more valuable products ${ }^{16}$ through chemical methods. Common chemical methods for recycling waste PET include methanolysis ${ }^{17-20}$, glycolysis ${ }^{21-25}$, neutral hydrolysis $^{26-29}$, alkaline hydrolysis ${ }^{30-32}$, acidic hydrolysis ${ }^{33-37}$, etc (Table 1). However, the monomers or oligomers obtained by methanolysis or glycolysis are difficult to separate and purify, which makes the above methods highly sensitive to the source of waste PET. Besides, both acid and alkaline hydrolysis use strong acids or strong bases as solvents, which will cause serious corrosion to the equipment and produce a large amount of waste in the post-processing stage. Accordingly, due to the inadequacy of existing chemical recycling methods, colored or blended PET is regarded as "unrecyclable" back into high-quality, food-grade PET for upcycling ${ }^{38,39}$. Hence, it is of great practical significance to develop a cost-effective and green method to realize the upcycling of waste PET.

Herein, we developed a new strategy to decompose waste PET into terephthalic acid (TPA) and ethylene glycol diacetate (EGDA) through acetolysis for upcycling. In this strategy, acetic acid has three main advantages: 1) Since the solubility of TPA in acetic acid is very low $\left(1.08 \times 10^{-2} \mathrm{~g} / 100 \mathrm{~g} @ 32^{\circ} \mathrm{C}, 3.86 \mathrm{~g} / 100 \mathrm{~g} @ 240^{\circ} \mathrm{C}\right)$, which not only ensures the 
or decompose other common components in PET materials (such as dyes, additives, blending ingredients, etc.) to ensure the separation of TPA from these impurities. 3) As the solvent for industrial oxidation of $p$-xylene to produce TPA ${ }^{40}$, acetic acid can be directly applied to existing production devices. Additionally, to compare acetolysis with other existing chemical recycling, we designed a path to complete the closed-loop recycling PET. Through the life-cycle assessment (LCA), the results show that acetolysis can become the lowest environmental impact of all chemical recycling options available today.

\begin{tabular}{|c|c|c|c|c|c|c|}
\hline \multirow{2}{*}{ Content } & \multirow{2}{*}{ Methanolysis $^{\text {a }}$} & \multirow{2}{*}{ Glycolysis } & \multicolumn{3}{|c|}{ Hydrolysis } & \multirow{2}{*}{$\begin{array}{c}\text { Acetolysis } \\
\text { (this work) }\end{array}$} \\
\hline & & & Neutral & alkaline & Acid & \\
\hline Pressure & $10-20 \mathrm{MPa}$ & $0.1 \mathrm{MPa}$ & $2-40 \mathrm{MPa}$ & 1-2 $\mathrm{MPa}$ & $0.1-2.8 \mathrm{MPa}$ & 1.2-3.2 MPa \\
\hline Temperature & $200-350^{\circ} \mathrm{C}$ & $150-200^{\circ} \mathrm{C}$ & $250-400^{\circ} \mathrm{C}$ & $80-250^{\circ} \mathrm{C}$ & $80-230^{\circ} \mathrm{C}$ & $220-280^{\circ} \mathrm{C}$ \\
\hline Products & $\begin{array}{l}\text { DMT } \\
\text { EG }\end{array}$ & $\begin{array}{c}\text { BHET } \\
\text { Oligomers }\end{array}$ & $\begin{array}{l}\text { TPA } \\
\text { EG }\end{array}$ & $\begin{array}{l}\text { TPA salt } \\
\text { EG }\end{array}$ & $\begin{array}{l}\text { TPA } \\
\text { EG }\end{array}$ & $\begin{array}{c}\text { TPA } \\
\text { EGDA }\end{array}$ \\
\hline Degradation & Completely & Incomplete & Incomplete & Complete & Incomplete & Complete \\
\hline Purification & Needed & Difficult & Complex & Complex & Complex & Easy \\
\hline Sensitivity $^{b}$ & High & High & Medium & Medium & Low & Low \\
\hline Concentration ${ }^{c}$ & $1-5 \% \mathrm{w} / \mathrm{v}$ & $2-25 \% \mathrm{w} / \mathrm{v}$ & $1-5 \% \mathrm{w} / \mathrm{v}$ & $1-5 \% \mathrm{w} / \mathrm{v}$ & $0.8-2 \% \mathrm{w} / \mathrm{v}$ & $20 \% \mathrm{w} / \mathrm{v}$ \\
\hline
\end{tabular}

\section{Results}

Acetolysis of PET plastics. As the solvent used in the industrial production of TPA, acetic acid was selected and it was more effective in depolymerizing PET than other carboxylic acids (Fig. 1a \& Table S1). Through acetolysis, PET could be completely depolymerized in two hours at $280^{\circ} \mathrm{C}$, and high-purity TPA $(95.8 \%$ yield, over $99.7 \%$ purity) and EGDA $(95.3 \%$ yield, over $98.0 \%$ purity) could be obtained. Our study found that the acetolysis of PET was very sensitive to the decrease of the reaction temperature (Fig. $1 \mathrm{~b} \&$ Table S2). When the temperature was below $220^{\circ} \mathrm{C}$, satisfactory results could not be obtained even if the reaction time was extended to 18 hours. We speculated that the depolymerization reaction undergoes a melting-dissolving process, so $280^{\circ} \mathrm{C}$ was chosen as the optimal reaction temperature. It is worth mentioning that the acetolysis of PET is an exothermic reaction (Fig. 1d). A little energy is needed to maintain the reaction temperature, so the acetolysis of PET is not a high energy consumption process. Additionally, a certain amount of water will not have adverse 
1c \& Table S3). This showed that the process did not have strict requirements for water in the pretreatment stage.

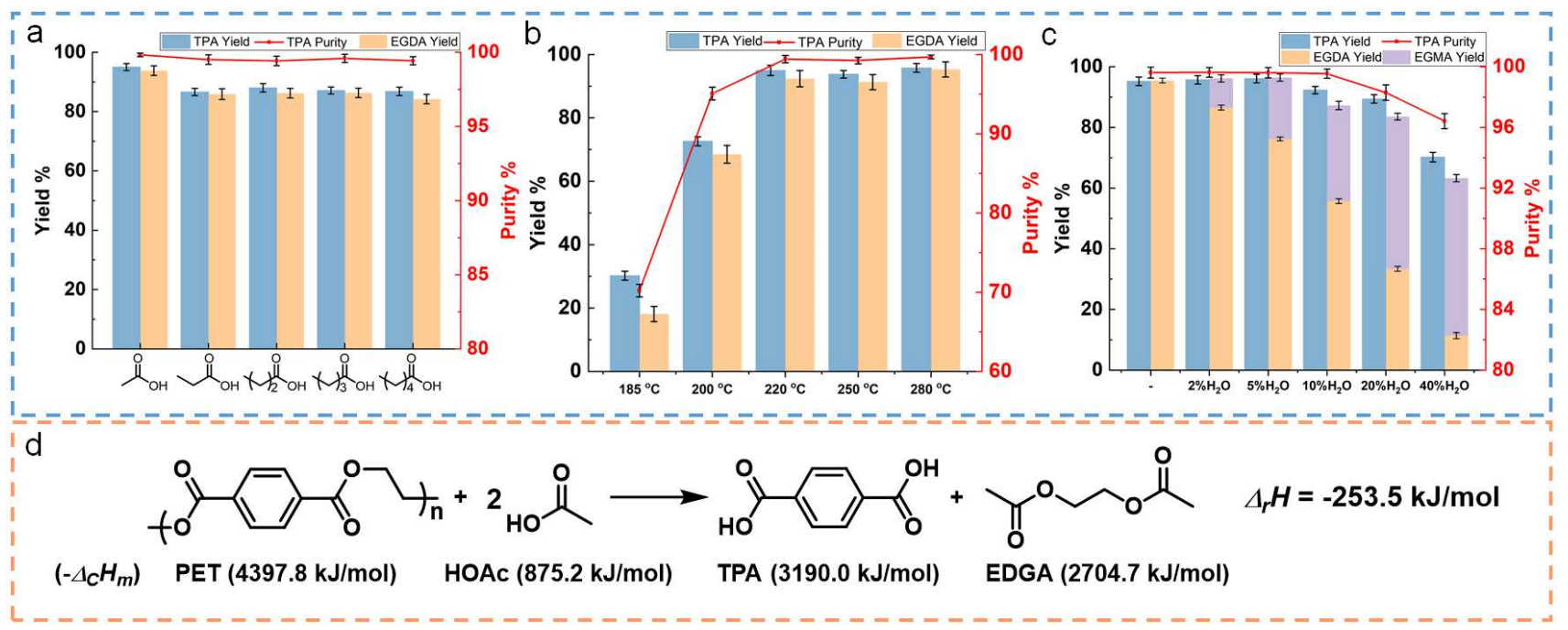

Fig. 1 Acetolysis of PET. a, Effects of different carboxylic acids on the acetolysis of PET b, Effects of different temperatures on the acetolysis of PET. c, Effects of different moisture contents on the acetolysis of PET. d, Reaction enthalpy of the acetolysis of PET.

To better understand the depolymerization behavior of PET in carboxylic acid, a surveillance video was recorded (see an evenly dispersed emulsion. Then the melted PET depolymerized into small molecular weight oligomers that could be dissolved in carboxylic acids. Finally, the oligomers further depolymerized to form TPA and crystallized out in the system. XRD (Fig. 2b-2c and Extended Data Fig. 1). This process, unlike hydrolysis, was particularly efficient as the depolymerization of oligomers dissolved in carboxylic acid was a homogeneous reaction. The precipitation of TPA not only effectively drives the chemical equilibrium of depolymerization, but also makes the separation of products simpler. 
$76 \mathrm{~GB}^{42}$ and the $\mathrm{ASTM}^{43}$ standard for polymerization grade TPA. These guaranteed the purity of TPA obtained by acetolysis

77 of PET and it also could be proved by titration analysis, HPLC, FTIR, UV-Vis, etc. (Extended Data Fig. 1).

Fig. 2e illustrated that acetolysis had a strong tolerance to the source of waste PET plastics (please see Table S5 for

79 more examples). PET plastics like powder, particles or bottle flakes (colorless, colored or even black) could be quantitatively

80 deagglomerated into high purity TPA and EGDA. For PET flakes mixed with polyethylene (PE) labels and PET-

81 polypropylene (PP) composite films, PET decomposed into TPA sinking, whereas PE and PP melted, agglomerated, and

82 floated on the solution after acetolysis (Extended Data Fig. 2). However, with a similar density to PET, polyvinyl chloride

83 (PVC) will be partially decomposed but not affect the depolymerization of $\mathrm{PET}^{44}$. In addition, other polyester plastics such

84 as Poly (ethylene terephthalateco-1,4-cylclohexylenedimethylene terephthalate) (PETG), polybutylene terephthalate (PBT),

85 Polyethylene naphthalenediate (PEN), and Polyethylene 2,5-furandicarboxylate (PEF) could be degraded by this process

86 with corresponding monomers obtained in high yield (Table S7). 


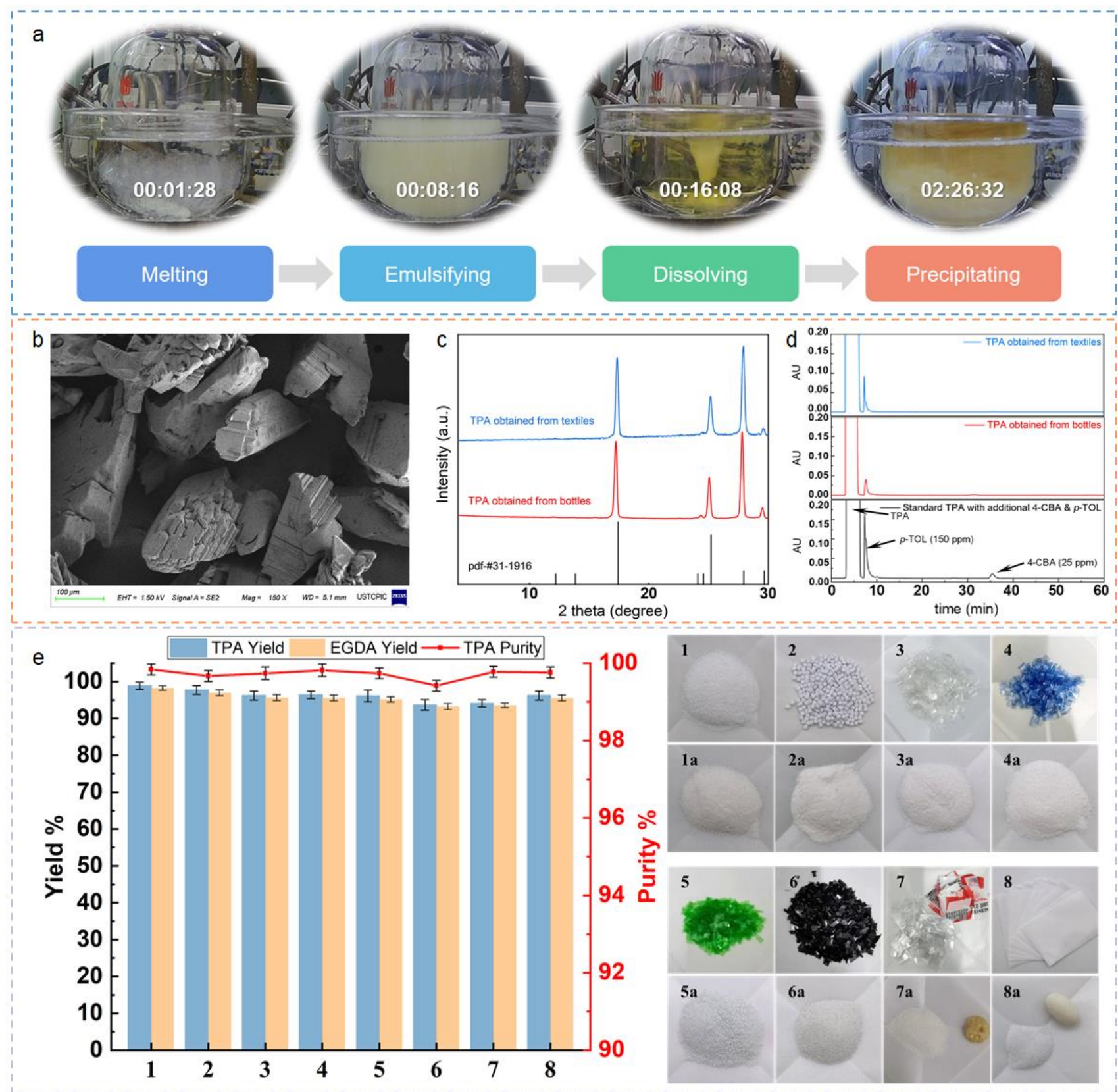

Fig. 2 Results of chemical degradation of PET through acetolysis. a, the reaction process of acetolysis of PET bottles. b, SEM pattern of TPA obtained from waste PET bottles through acetolysis. c, XRD pattern of TPA obtained from waste PET bottles and textiles, d, HPLC spectra of TPA obtained from waste PET bottles and textiles. e, Universality of PET degraded by acetolysis, 1-8 are photos of PET powder, particles, transparent bottle, blue bottle, green bottle, black tray, physical mixing of PET and PP, PET/PP composite films, 1a-8a are the photos of terephthalic acid obtained by acetolysis of the above materials.

Acetolysis of PET textiles. PET fiber, namely terylene, is the highest market shared chemical fiber with the most important application of PET products (ca. 80\%). Compared with bottle grade PET, fiber grade PET generally has a lower degree of polymerization, and its textiles are generally more complex. For PET textiles, the current physical recycling method can 
only be downcycling since the gradually decreasing degree of polymerization, while the chemical recycling method is unable to achieve upcycling due to the difficulty of separating and purifying the depolymerized products.

We applied the acetolysis method to successfully achieve the depolymerization of PET textiles (Table 2, please see

99 Extended Data Fig. 3 and 4 for more examples). Since some dyes or other organic impurities are partially decomposed and

100 carbonized during the acetolysis process, the depolymerized product TPA resulted in a darker color. These dark TPAs could

101 be decolorized by activated carbon after being dissolved in alkaline or amide (e.g.: N, N-dimethylacetamide, N-methyl

102 pyrrolidone). Processing the above decolorization, white PET with qualified chromaticity (Hazen $<10)$ could be obtained ${ }^{42}$.

103 Even altering the high-temperature $\left(280^{\circ} \mathrm{C}\right)$ pure water recrystallization with activated carbon to decolor, the appearance

104 of the product can also be significantly improved (Extended Data Fig. 5).

In addition, for PET blended textiles, the method of acetolysis is also applicable. Since acetic acid can decompose and

106 transform other common chemical fibers at high temperatures to form acetic acid-soluble depolymerization products (e.g.:

107 nylon 6 is decomposed into acetaminocaproic acid; nylon 66 is decomposed into adipic acid and diacetyl hexamethylene

108 diamine; acrylic fiber is converted into polyacrylic acid; spandex is decomposed into acetanilide), so it will not affect the separation of TPA. Meanwhile, because these chemical fiber decomposition products have high boiling points, this will not

110 cause the separation and purification of EGDA via distillation to be more troublesome. It is worth mentioning that EGDA

111 is non-toxic and has a strong dissolving ability that can be used as a green solvent for ink coatings, making this acetolysis

112 process more economical. 
Table 2. Degradation of real textiles by acetolysis ${ }^{\text {a }}$

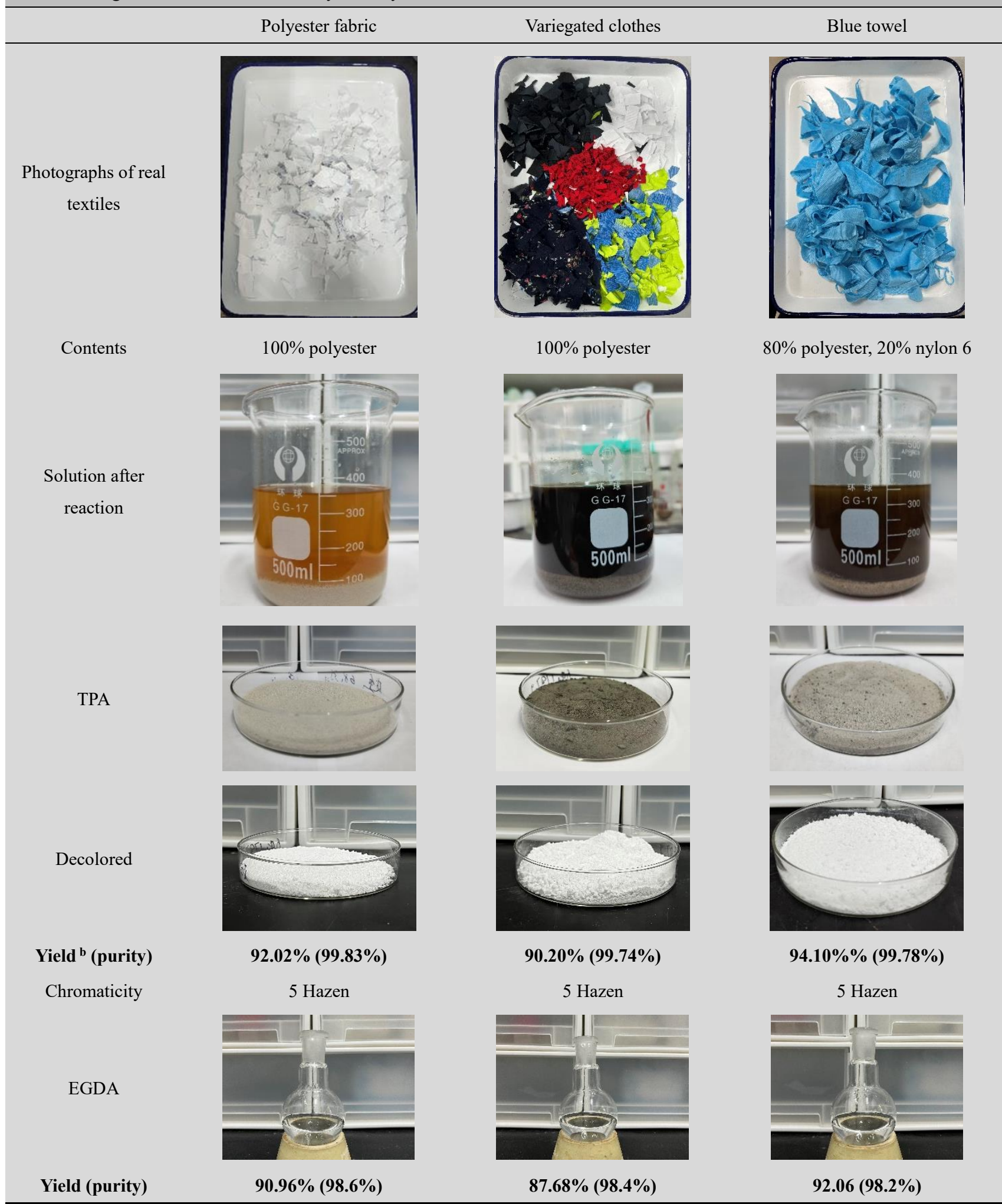

${ }^{\text {a }}$ Reaction conditions: PET Textile fragments $(60 \mathrm{~g})$, acetic acid $(300 \mathrm{~mL})$, reaction temperature $\left(280^{\circ} \mathrm{C}\right)$, reaction time $(2 \mathrm{~h})$.

${ }^{\mathrm{b}}$ Yields are calculated for decolorized TPA based on PET textile raw materials 
113 Life-cycle assessment for closed-loop upcycling PET via acetolysis process. We implemented a life-cycle assessment

114 (LCA) study to compare and evaluate the environmental impact degree of the acetolysis method with other existing

115 methods for recycling PET. Since the subsequent use and disposal of various PET products vary greatly, the system

116 boundary of this LCA is set as "cradle-to-gate". To make a fair comparison with previous work and to simplify the

117 analysis ${ }^{46-49}$, the LCA of PET recycling is based on the "cut-off" approach (Fig. 3a). According to the above rules, we

118 assumed that the difference of environmental impact to the collection and transportation of various waste PET materials

119 could be negligible, then the scope of this LCA was "gate-to-gate". Within the "gate-to-gate" boundary, the mechanical

120 recycling includes two units, "waste PET shredding" and "PET flakes thermoforming", while the chemical recycling adds

121 a "depolymerization \& re-polymerization" unit. Therefore, as a chemical recovery method, we focused on the

122 environmental impact of the acetolysis process in "gate-to-gate".

The process for upcycling PET from waste PET via acetolysis was shown in Fig. 3b, which includes five units: 1)

124 mechanical shredding and other simple pretreatments of waste PET; 2) acetolysis of PET flakes to produce TPA and EGDA

125 (Section1); 3) hydrolysis of EGDA into ethylene glycol (EG)(Section2); 4) re-polymerizing of EG and TPA into PET resins

126 (Section3); and 5) thermoforming of PET resins into products. The first and last two units could be attributed to mechanical

127 recycling. The process composed of the remaining three units (Section 1-3) was simulated on an industrial scale (100,000

128 tons per year) in ASPEN Plus V11 in terms of mass balance and energy consumption. The global warming potential (GWP)

129 and non-renewable energy use (NREU) of the process were calculated by OpenLCA. 

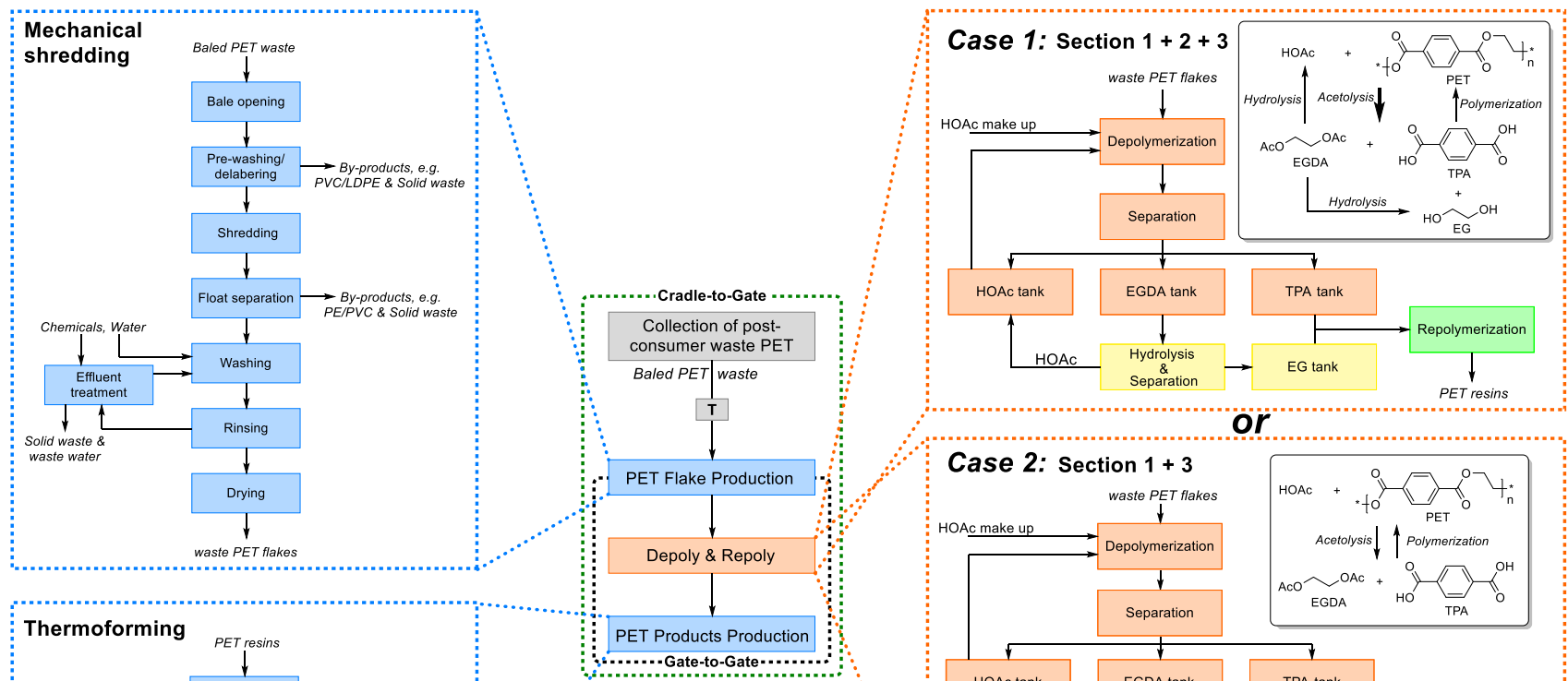

Or
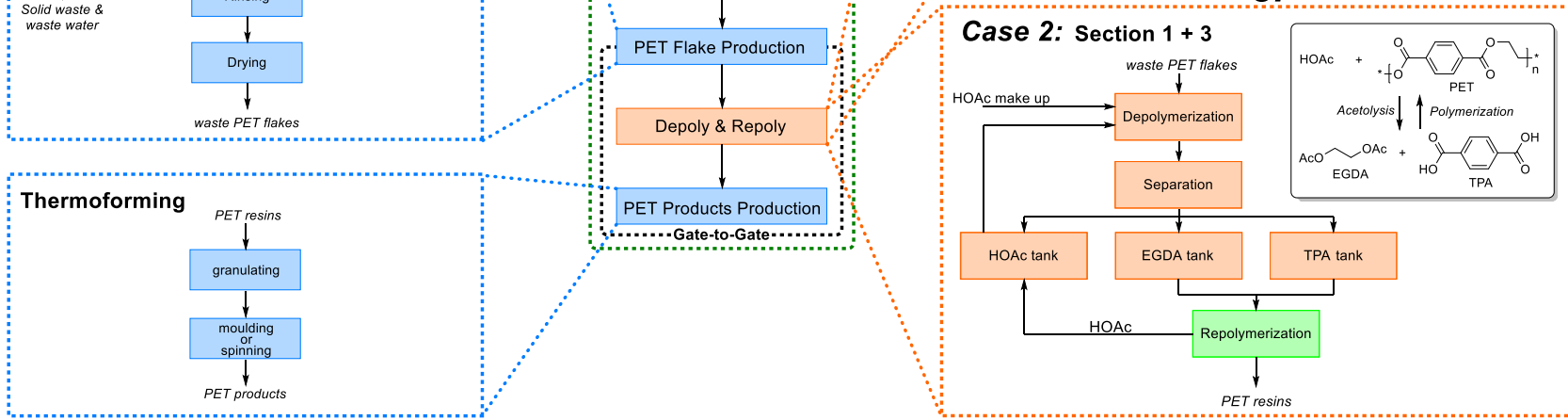

PET Products Production

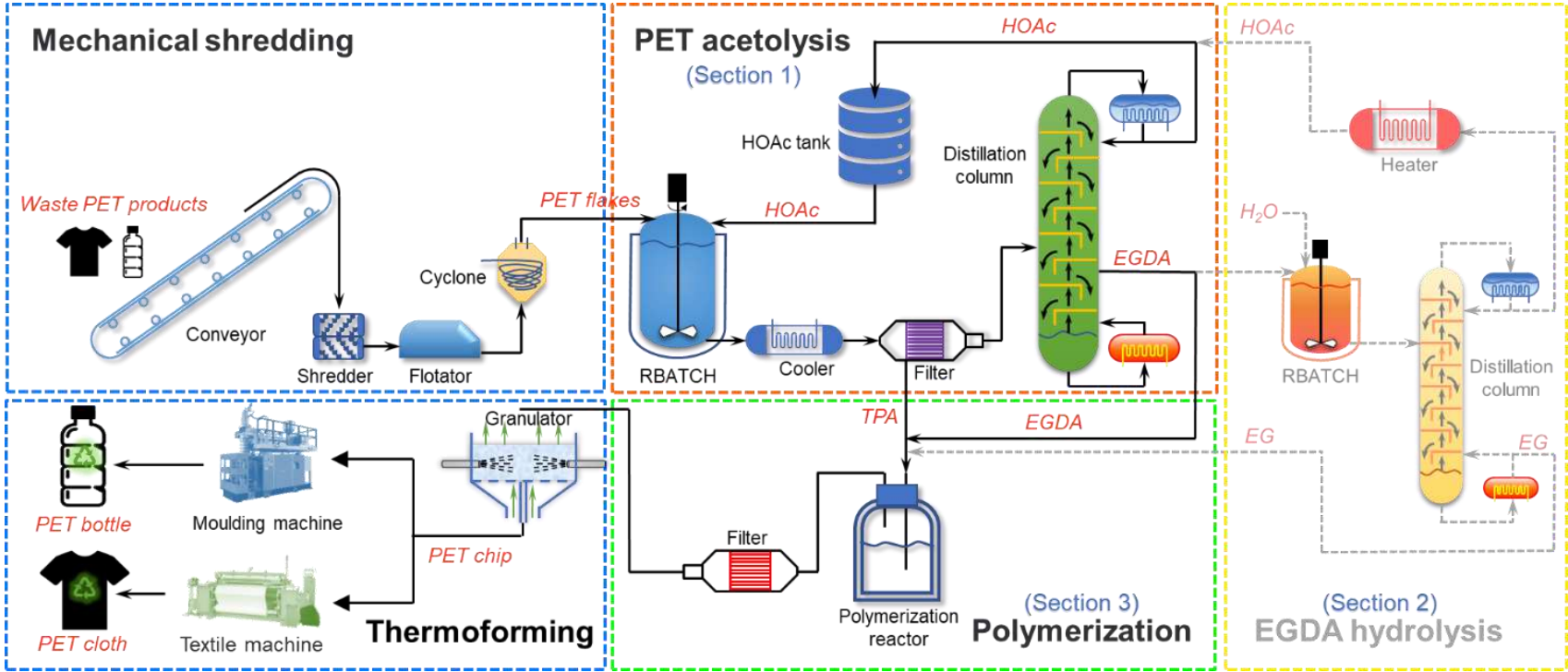

Fig. 3 the process for closed-loop upcycling PET via acetolysis. a, Cradle-to-factory gate system boundary of closed-loop upcycling

PET via an acetolysis process based on the "cut-off" approach. b, Schematic diagram of closed-loop upcycling PET via the acetolysis.

LCA study data illustrated that, compared to the process for preparing virgin-PET by the fossil resource ${ }^{46}$, the GWP and NREU of the acetolysis process (Case 1 of Figure 3a) could be reduced by $26 \%$ and $47 \%$ respectively, which was very close to the methanolysis process (Fig. 4a). Further sensitivity analysis showed that section 2 (the EGDA hydrolysis section) contributed the most GWP and NREU (Fig. 4b). This was mainly because a large amount of water was needed to ensure complete hydrolysis of EGDA, while the separation of EG, acetic acid, and water required a lot of energy. Therefore, a low 
139 energy consumptive process for separating and purifying the hydrolysate EG was desired. In addition, we proposed and

140 assessed another process that omits Section 2 (Case 2 of Figure 3a). In this process, EGDA would be directly condensed

141 with TPA and polymerized into PET by removing acetic acid. Due to the omission of the hydrolysis section, LCA results

142 showed that PET produced in a closed-loop cycle via case 2 could reduce GWP (1.35 $\mathrm{kg} \mathrm{CO}$-equivalent/kg PRO-PET)

143 and NREU (22 MJ/kg PRO-PET). This is the lowest environmental impact degree in chemical degradation at present. The

144 specific process, energy consumption, and material balance of case 1 and case 2 were described in supplementary Fig. S2-

145 S3, supplementary table S12-S19, and supplementary Note 2-3 respectively. Besides, this process has the potential

146 advantage of not forming diethylene glycol fragments, which usually affect the strength of PET, on the polyester chain.

147 However, since the nucleophilicity of the acetoxy group is weaker than that of the hydroxyl group, it is necessary to seek

148 a more effective catalyst to promote the polymerization of EGDA and TPA. Despite this, the acetolysis of PET we proposed

149 is currently the only method that can obtain high-purity TPA directly from waste PET. This method provides a feasible and

150 low environmental impact solution for upcycling waste PET.

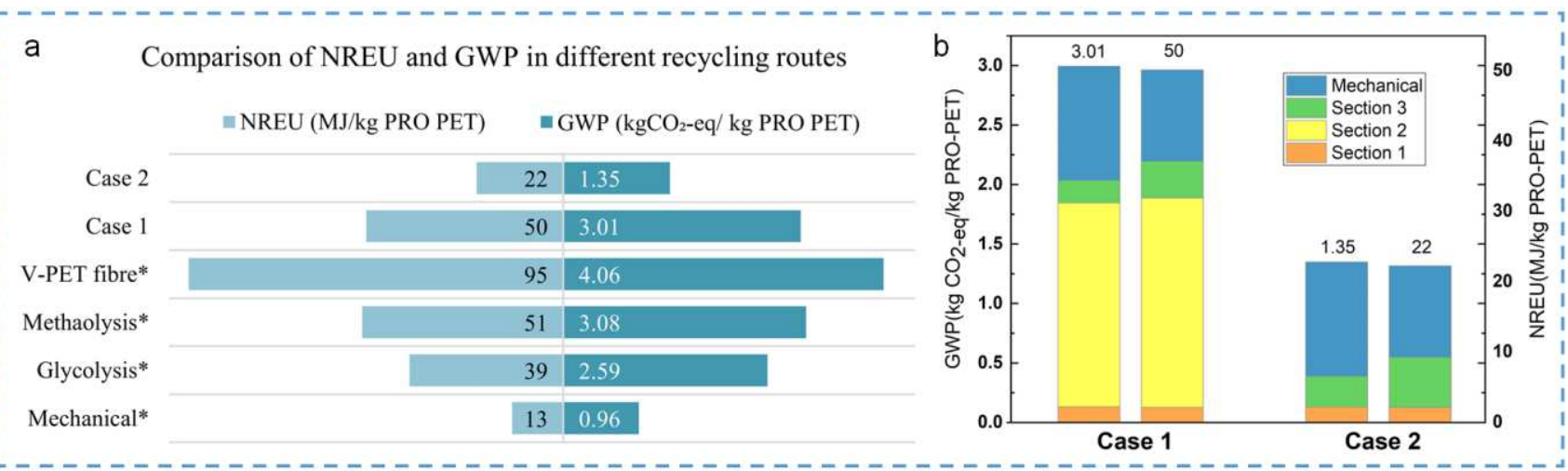

15

152 Fig. 4 LCA results for closed-loop upcycling PET via acetolysis. a, LCA results for $1 \mathrm{~kg}$ of recycled PET, based on the "cut off" approach, cradle-to-factory gate. ${ }^{*}$ The data is from Ref. $46 . \mathbf{b}$, Sensitivity analysis of NREU and GWP via acetolysis.

\section{Discussion}

In this work, we developed a simple and cost-effective method for the upcycling of waste PET bottles and textiles by 
157 And the acetolysis is compatible with impurities, dyes, and other components (polyethylene or polypropylene or nylon, etc.) present in waste PET. We have also proposed two schemes for closed-loop recycling of PET via acetolysis and assessed their environmental impact through LCA. LCA results showed that the production of PET by acetolysis could significantly reduce the environmental impact in terms of GWP and NREU compared to conventional petroleum routes. Under the optimal conditions, acetolysis has the lowest environmental impact of the currently available chemical recycling methods for waste PET. Besides, as a new generation of green solvents, the by-product EGDA brings greater economic attractiveness to acetolysis. Briefly, this work provides a promising and sustainable way for the upcycling of waste PET materials.

\section{Methods}

Polyester materials, chemicals, and reagents. In all experiments, PET fragments were obtained from various PET

products, including transparent and colored, mono- and multi-layer post-consumer PET lunch boxes and trays, and PET water bottles without caps and labels. These materials were simply washed with tap water, dried, and cut into approximately $3 \times 3 \mathrm{~mm}$ fragments for experiments. PET textiles fragments were donated by the author and his collaborators. PEF particles (yellow solid particles, average molecular weight $>30000$ ) and PEN polyester (pressure-sensitive silicone tape) were generously gifted by Hefei Leaf Biotechnology Co., Ltd. PETG polyester (3D printing consumables) was purchased in the Lambo flagship store. These materials were cut into fragments (or particles). PBT powder (300 mesh) was purchased from the Hongyuan polymer supply chain. PVC powder was purchased from Suyuan plastic raw material mall. TPA(99\%), p-TOL (98\%), 4-CBA (98\%) were purchased from Adamas Reagent Co., Ltd. Other chemicals are commercially available and utilized without further treatment.

175 Acetolysis of PET plastics. A typical acetolysis process was shown as follows. $60.0 \mathrm{~g}$ PET flakes and $300 \mathrm{~mL}$ glacial acetic acid were mixed in a $500 \mathrm{~mL}$ titanium hydrothermal reactor. Then the hydrothermal reactor was placed in a homogeneous reactor for depolymerization at a constant temperature of $280^{\circ} \mathrm{C}$ for 2 hours. After the reaction, the hydrothermal reactor was cooled down to room temperature. The solid products obtained by filtration and were washed 
three times with pure water and dried in an oven. The TPA yield was calculated by equation (1). The purity of TPA was tested by acid-base titrations according to the National Standard of the People's Republic of China GB/T 30921.5-2016.

$$
\begin{gathered}
\text { TPA Yield (\%) }=\frac{\text { Produced TPA amount }(\mathrm{g})}{\text { Theoretically Produced TPA amount }(\mathrm{g})} \times 100(\%) \\
=\frac{\text { TPA qunality by obtained }(\mathrm{g})}{\frac{\text { PET amount }(\mathrm{g})}{192.13\left(\frac{\mathrm{g}}{\mathrm{mol}}\right)} \times 166.13\left(\frac{\mathrm{g}}{\mathrm{mol}}\right)} \times 100(\%)
\end{gathered}
$$

The products in the liquid phase were analyzed by GC equipped with a flame ionization detector (FID) for quantitative

analysis. The yield of EGDA was calculated by equation (2):

$$
\begin{gathered}
\text { EGDA Yield }(\%)=\frac{\text { Produced EGDA amount }(g)}{\text { Theoretically Produced EGDA amount }(g)} \times 100(\%) \\
=\frac{\text { EGDA amount qunatified by GC }(\mathrm{g})}{\frac{\text { PET amount }(\mathrm{g})}{192.13\left(\frac{\mathrm{g}}{\mathrm{mol}}\right)} \times 146.14\left(\frac{\mathrm{g}}{\mathrm{mol}}\right)} \times 100(\%)
\end{gathered}
$$

Acetolysis of PET textiles. A typical acetolysis for textiles process was shown as follows. $60.0 \mathrm{~g}$ PET textile fragments and $300 \mathrm{~mL}$ glacial acetic acid were mixed in a $500 \mathrm{~mL}$ titanium hydrothermal reactor. Then the hydrothermal reactor was placed in a homogeneous reactor for depolymerization at a constant temperature of $280^{\circ} \mathrm{C}$ for 2 hours. After the reaction, the hydrothermal reactor was cooled down to room temperature. The products were separated and analyzed as previously shown.

Decolorization of dark TPA. Decolorization of black TPA was carried out by activated carbon. a) $40 \mathrm{~g}$ of dark TPA and $0.4 \mathrm{~g}$ of activated carbon were added to a $200 \mathrm{~mL}$ aqueous solution containing $20 \mathrm{~g}$ of sodium hydroxide and the mixture was refluxed for 15 minutes. The impurities were removed by hot filtration and white TPA was obtained by adding an equal amount of dilute sulphuric acid. b) $40 \mathrm{~g}$ of dark TPA and $0.4 \mathrm{~g}$ of activated carbon were added to $200 \mathrm{~mL}$ of N, Ndimethylacetamide (DMAC) and the mixture was stirred at $80^{\circ} \mathrm{C}$ for 15 minutes. The insoluble impurities were removed by hot filtration. The adductive crystals of TPA and DMAC selectively precipitated out of the filtrate. White TPA could be obtained by heating the crystals or adding water after filtration. c) $40 \mathrm{~g}$ of dark TPA and activated carbon fiber felt $(10 \mathrm{~cm}$ $\times 15 \mathrm{~cm}$ ) were added to $200 \mathrm{~mL}$ of water and stirred for 15 minutes at $280^{\circ} \mathrm{C}$. Then cooled the reactor to room temperature slowly to obtain clean TPA crystals. All dark products obtained by acetolysis of waste PET materials were easily decolorized in the above described ways. 
204 Characterization. Products in the liquid phase were detected by flame ionization detector gas chromatography (GC-FID, 205 Shimadzu-Nexis GC-2030), which used HP-INNOWAX $(30 \mathrm{~m} \times 0.25 \mathrm{~m} \times 0.25 \mathrm{~mm})$ as a capillary column. High206 performance liquid chromatography (HPLC) was performed using Agilent HC-C18, 4.6×250mm column and Waters UV 207 detector 2489 at $254 \mathrm{~nm}$. Nuclear magnetic resonance (NMR) spectroscopic measurements were carried out in deuterated 208 dimethyl sulfoxide (DMSO-d6) or deuterated chloroform $\left(\mathrm{CDCl}_{3}\right)$ on a Bruker Advance $400(400 \mathrm{MHz})$ spectrometer for 209 the product characterization. The morphology of the samples was observed with Zeiss Gemini 500 Schottky field emission 210 gun scanning electron microscope (SEM) (2-5kV). X-ray powder diffraction (XRD) data were acquired on a JEOL-2010 211 diffractometer using $\mathrm{Cu} \mathrm{K} \alpha$ radiation at 4 30 degrees. Fourier transform infrared spectrum (FTIR) were recorded on an 212 FTIR/Raman Thermo Nicolet 8700 spectrometer in the wavenumber range of 3800 to $500 \mathrm{~cm}^{-1}$, with instrument resolution 213 of $0.1 \mathrm{~cm}^{-1}$. The Ultra Violet-Visible Spectroscopy (UV-VIS) absorption spectra were collected with a UV-1750 UV-vis 214 spectrophotometer (Shimadzu, Japan).

215 Life-cycle assessment. The LCA analysis followed the ISO standard series 14040 and was conducted using OpenLCA 216 1.10.3. Two functional units were used, one was a constant mass $(1 \mathrm{~kg})$ of the new PET plastic produced and the other was 217 the standardization of the environmental impact results through the production of PET by acetolysis, which enabled us to 218 compare different methods of PET recycling in terms of environmental impact. The system boundaries were set as "cradle219 to-gate", which included: collection and transportation of waste PET, production of PET flakes, the whole process of 220 depolymerization and re-polymerization, and PET products thermoforming. See details in the Supplementary Information.

\section{Data availability}

222 Data are available on reasonable request from the authors, according to their contributions.

\section{References}

2241 Smith, J. \& Vignieri, S. A devil's bargain. Science 373, 34-35 (2021).

2252 Plastic upcycling. Nature Catalysis 2, 945-946 (2019).

2263 Geyer, R., Jambeck, J. R. \& Law, K. L. Production, use, and fate of all plastics ever made. Sci Adv 3 (2017).

2274 Uekert, T., Kasap, H. \& Reisner, E. Photoreforming of Nonrecyclable Plastic Waste over a Carbon Nitride/Nickel 228 Phosphide Catalyst. J Am Chem Soc 141, 15201-15210 (2019).

2295 Barnard, E., Rubio Arias, J. J. \& Thielemans, W. Chemolytic depolymerization of PET: a review. Green Chemistry 23, 3765-3789 (2021). 
2316 Rahimi, A. \& García, J. M. Chemical recycling of waste plastics for new materials production. Nature Reviews 232 Chemistry 1, 0046 (2017).

2337 Wei, R. et al. Biocatalytic Degradation Efficiency of Postconsumer Polyethylene Terephthalate Packaging 234 Determined by Their Polymer Microstructures. Adv Sci (Weinh) 6, 1900491 (2019).

2358 Tournier, V. et al. An engineered PET depolymerase to break down and recycle plastic bottles. Nature 580, 216$236219(2020)$.

2379 Chen, C.-C. et al. General features to enhance enzymatic activity of poly(ethylene terephthalate) hydrolysis. $238 \quad$ Nature Catalysis 4, 425-430 (2021).

23910 Joo, S. et al. Structural insight into molecular mechanism of poly(ethylene terephthalate) degradation. Nat $240 \quad$ Commun 9, $382(2018)$.

241 Thomas, K. V. Understanding the plastics cycle to minimize exposure. Nature Sustainability, 242 doi:10.1038/s41893-021-00814-3 (2021).

$24312 \quad$ Ellis, L. D. et al. Chemical and biological catalysis for plastics recycling and upcycling. Nature Catalysis 4, 539$556(2021)$.

Sanchez-Rivera, K. L. et al. Reducing Antisolvent Use in the STRAP Process by Enabling a TemperatureControlled Polymer Dissolution and Precipitation for the Recycling of Multilayer Plastic Films. ChemSusChem 14, 4317-4329 (2021)

Walker, T. W. et al. Recycling of multilayer plastic packaging materials by solvent-targeted recovery and precipitation. Sci Adv 6, eaba7599 (2020).

Zhang, F. et al. Polyethylene upcycling to long-chain alkyl aromatics by tandem hydrogenolysis/aromatization. Science 370, 437-441 (2020).

Zhou, H. et al. Electrocatalytic upcycling of polyethylene terephthalate to commodity chemicals and $\mathrm{H}_{2}$ fuel. $\mathrm{Nat}$ Commun 12, 4679 (2021).

Genta, M., Iwaya, T., Sasaki, M., Goto, M. \& Hirose, T. Depolymerization mechanism of poly(ethylene terephthalate) in supercritical methanol. Industrial \& Engineering Chemistry Research 44, 3894-3900 (2005). in supercritical methanol. Journal of Physics: Condensed Matter 14, 11427-11430 (2002).

Yang, Y., Lu, Y. J., Xiang, H. W., Xu, Y. Y. \& Li, Y. W. Study on methanolysis depolymerization of PET with supercritical methanol for chemical recycling. Polymer Degradation and Stability 75, 185-191 (2002). 

the presence of aluminum triisopropoxide catalyst to form dimethyl terephthalate and ethylene glycol. Polymer Degradation and Stability 79, 529-533 (2003). Jehanno, C. et al. Organocatalysed depolymerization of PET in a fully sustainable cycle using thermally stable protic ionic salt. Green Chemistry 20, 1205-1212 (2018).

Delle Chiaie, K. R., McMahon, F. R., Williams, E. J., Price, M. J. \& Dove, A. P. Dual-catalytic depolymerization of polyethylene terephthalate (PET). Polymer Chemistry 11, 1450-1453 (2020).

Fang, P. et al. High-efficiency glycolysis of poly(ethylene terephthalate) by sandwich-structure polyoxometalate catalyst with two active sites. Polymer Degradation and Stability 156, 22-31 (2018).

Bahramian, A. The effect of thermal and non-thermal routes on treatment of the $\mathrm{Mg}-\mathrm{Al}$ layered double hydroxide catalyst dispersed by titania nanoparticles in products distribution arising from poly(ethylene terephthalate) degradation. Polymer Degradation and Stability 179, 109243 (2020).

Laldinpuii, Z., Lalhmangaihzuala, S., Pachuau, Z. \& Vanlaldinpuia, K. Depolymerization of poly(ethylene terephthalate) waste with biomass-waste derived recyclable heterogeneous catalyst. Waste Manag 126, 1-10 (2021)

Liu, Y., Wang, M. \& Pan, Z. Catalytic depolymerization of polyethylene terephthalate in hot compressed water. The Journal of Supercritical Fluids 62, 226-231 (2012).

Campanelli, J. R., Cooper, G. \& Kamal, M. R. Catalyzed-Hydrolysis of Polyethylene Terephthalate Melts. J Appl Polym Sci 53, 985-991 (1994).

28

Čolnik, M., Knez, Ž. \& Škerget, M. Sub- and supercritical water for chemical recycling of polyethylene terephthalate waste. Chemical Engineering Science 233,116389 (2021). hydrolysis. Green Chemistry 22, 5376-5394 (2020). estrutural do ácido tereftálico e efeito da hidrólise alcalina em baixa temperatura. Polímeros 24, 567-571 (2014). 

Roum Chim 57, 1031-1036 (2012).

29235 Wang, Y. et al. Zinc-catalyzed ester bond cleavage: Chemical degradation of polyethylene terephthalate. Journal of Cleaner Production 208, 1469-1475 (2019).

Mancini, S. D. \& Zanin, M. Post Consumer Pet Depolymerization by Acid Hydrolysis. Polymer-Plastics Technology and Engineering 46, 135-144 (2007).

Mishra, S., Goje, A. S. \& Zope, V. S. Chemical Recycling, Kinetics, and Thermodynamics of Hydrolysis of Poly(Ethylene Terephthalate) (PET) Waste in Sulfuric Acid in Presence of Phosphoric Acid. Polymer-Plastics Technology and Engineering 42, 581-603 (2007).

Rorrer, N. A. et al. Combining Reclaimed PET with Bio-based Monomers Enables Plastics Upcycling. Joule 3, 1006-1027 (2019).

Forrest, M,. Recycling of polyethylene terephthalate. (Smithers Rapra Technology Ltd. Press, 2016).

Li, M., Niu, F., Busch, D. H. \& Subramaniam, B. Kinetic Investigations of p-Xylene Oxidation to Terephthalic Acid with a $\mathrm{Co} / \mathrm{Mn} / \mathrm{Br}$ Catalyst in a Homogeneous Liquid Phase. Industrial \& Engineering Chemistry Research

53, 9017-9026 (2013).

Thomas, S. et al. Recycling of polyethylene terephthalate bottles. (Elsevier Inc. Press, 2019).

General Administration of Quality Supervision, Inspection and Quarantine of the People's Republic of China Standardization Administration of the People's Republic of China. Purified terephthalic acid(PTA)for industrial use: GB/T 32685-2016. Beijing: Standards Press of China, 2016.

American Society for Testing and Materials. Specification for purified terephthalic acid (PTA): ASTM D79762020. West Conshohocken: ASTM International, 2020.

Ellen MacArthur Foundation. New Plastics Economy: Rethinking the Future of Plastics \& Catalyzing Action. 2017. http://www.ellenmacarthurfoundation.org/publications.

Nagano, K. et al. Experimental studies on toxicity of ethylene glycol alkyl ethers in Japan. Environ Health Perspect 57, 75-84 (1984).

6 Shen, L., Worrell, E. \& Patel, M. K. Open-loop recycling: A LCA case study of PET bottle-to-fibre recycling. Resources, Conservation and Recycling 55, 34-52 (2010).

Semba, T., Sakai, Y., Sakanishi, T. \& Inaba, A. Greenhouse gas emissions of $100 \%$ bio-derived polyethylene terephthalate on its life cycle compared with petroleum-derived polyethylene terephthalate. Journal of Cleaner Production 195, 932-938 (2018). 

energy and GHG balance. Energy \& Environmental Science 5, 6407-6422 (2012). 767 (2020).

Acknowledgments

325 This work was financially supported by the National Natural Science Foundation of China (21875239). The authors thank 326 Hefei Leaf Biotech Co., Ltd and Anhui Kemi Machinery Technology Co., Ltd for free samples and equipment that benefited 327 their ability to conduct this study.

\section{Author Affiliations}

329 CAS Key Laboratory of Urban Pollutant Conversion, Anhui Province Key Laboratory of Biomass Clean Energy, 330 Department of Applied Chemistry, University of Science and Technology of China, Hefei, Anhui, 230026, PR China 331 Yuantao Peng, Jie Yang, Chenqiang Deng, Yao Fu, Jin Deng

\section{Author Contributions}

333 J. D. and Y. P. designed the experiments. Y. P. carried out experiments. J. Y. provided the LCAs. C. D. and Y. F. provided 334 some useful suggestions. J. D., Y. P. and J. Y. collaboratively analyzed the data and wrote the paper. All authors commented 335 on the final manuscript.

\section{Corresponding authors}

337 Correspondence to Jin Deng

338 Competing interests

339 The authors declare no competing interests. 

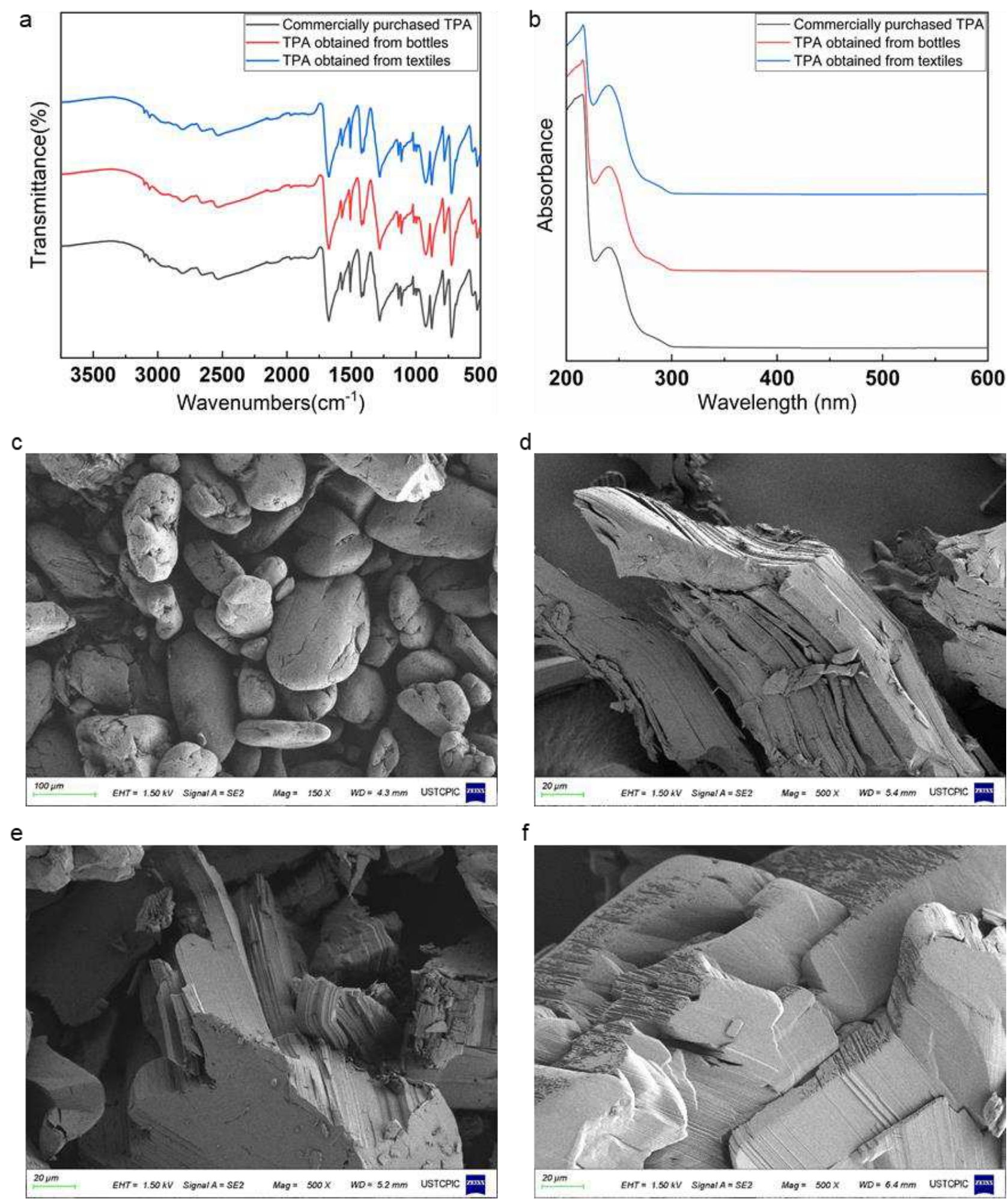

Extended Data Fig.1 Some spectral data of TPA obtained by acetolysis. a, FTIR spectra of TPA obtained from waste PET bottles and textiles. b, UV-Vis spectra of TPA obtained from waste PET bottles and textiles. c, TPA powder purchased commercially (50 - $200 \mu \mathrm{m})$. d, TPA obtained by acetolysis of PET bottle fragments $(500 X)$. e, TPA obtained by acetolysis of PET textiles fragments $(500 X)$. f, TPA obtained by acetolysis of PET powder $(500 X)$. It could be seen from FTIR and UV-Vis spectroscopy that the TPA obtained by acetolysis had high purity, whether it came from high-quality bottles or low-quality textiles. 

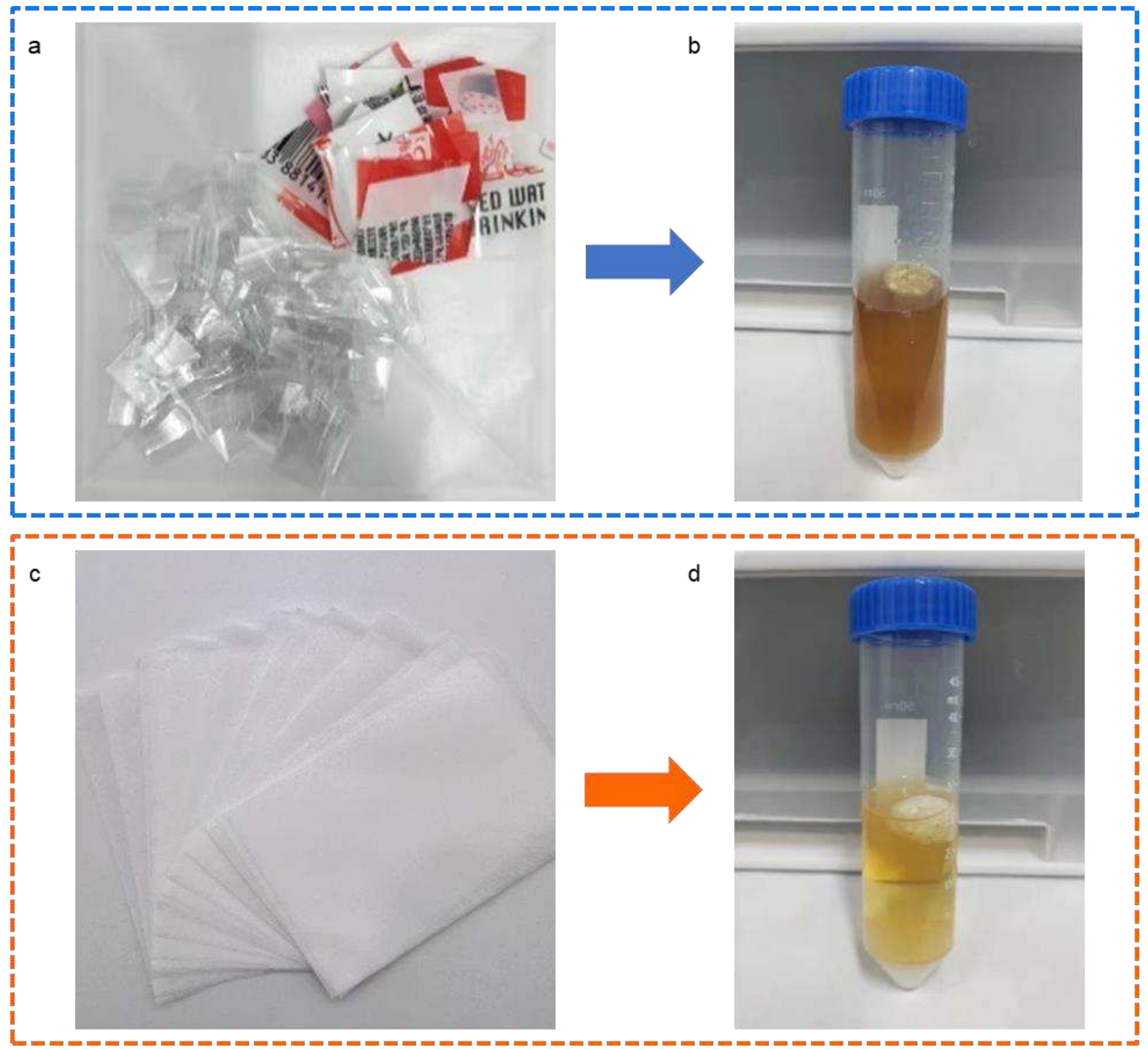

349 Extended Data Fig.2 Acetolysis of PET mixed with other plastics. a, Acetolysis of physically mixed flake PET with polyethylene

350 (PE) labels. b, the reaction solution after acetolysis, PET was decomposed into TPA and settled, while PE melted, clumps and floated on 351 the solution. $\mathbf{c}$, Acetolysis of PET-polypropylene (PP) composite films. d, the reaction solution after acetolysis, PET was decomposed into TPA and settled, while PP melted, clumped and floated on the solution. This meant that there was no need to separate the label for acetolysis of the PET bottles. 

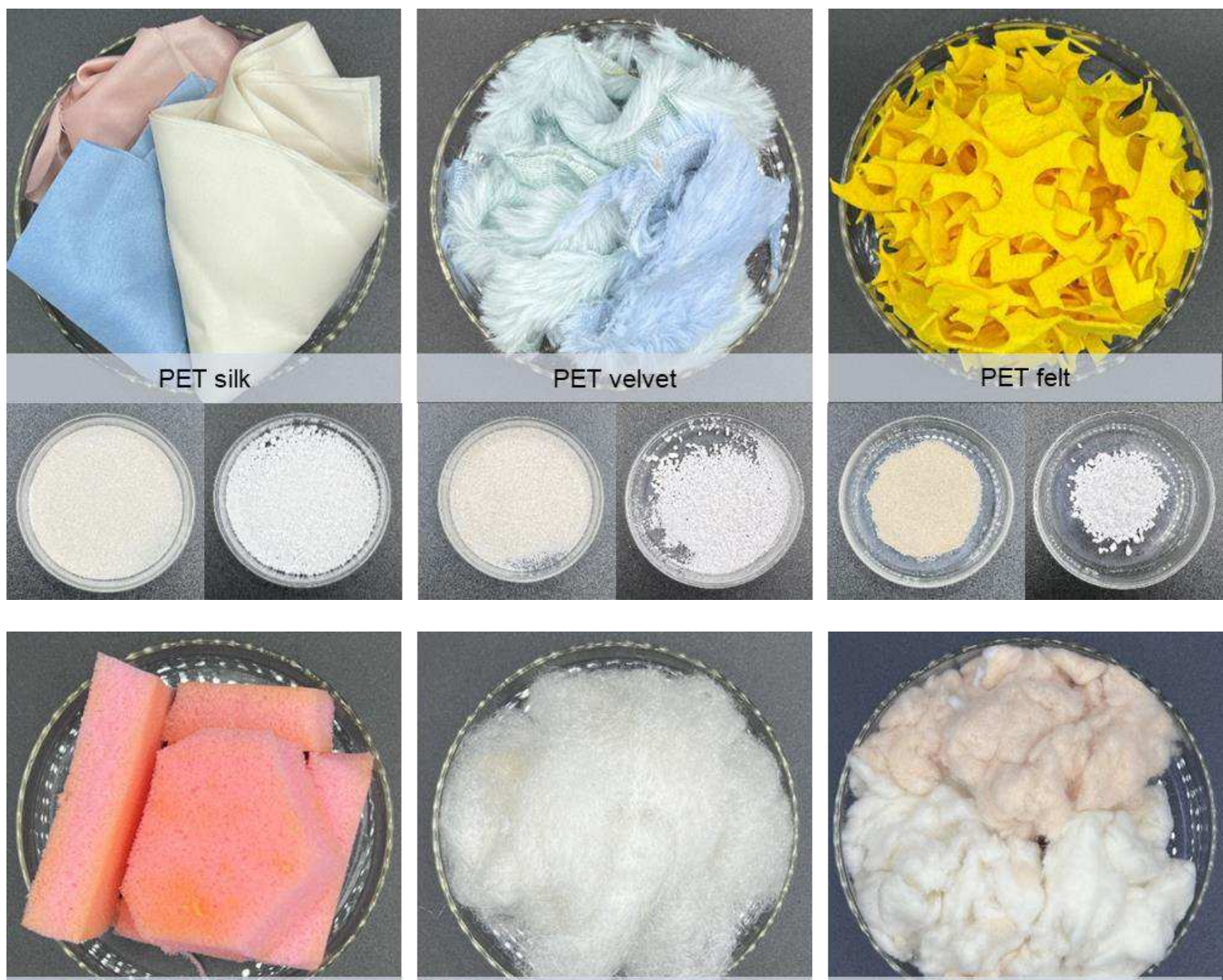

PET sponge
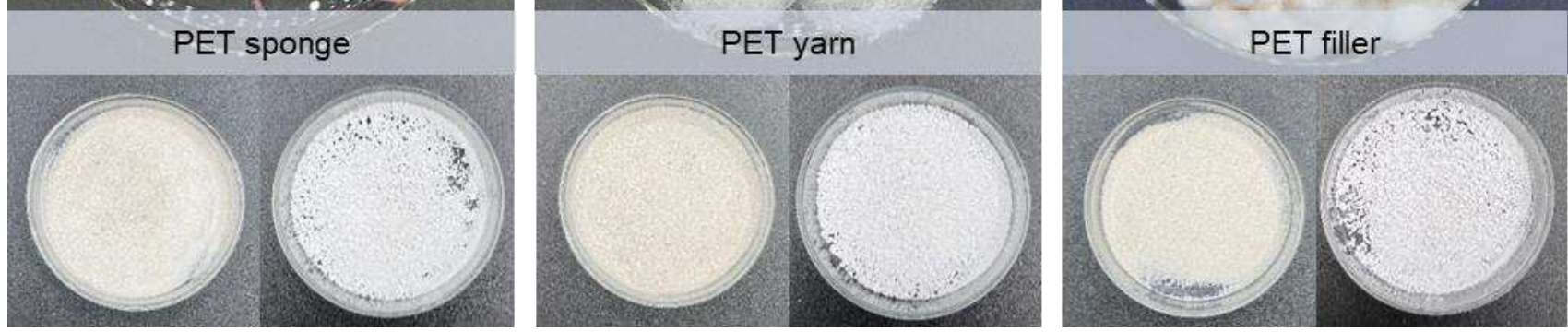

Extended Data Fig.3 Acetolysis of various PET fibers. Decolorized terephthalic acid obtained from different textiles yielded greater

than $90 \%$ and purity over $99.7 \%$. We tried the acetolysis of all PET fibers available and all of them could achieve good results. 


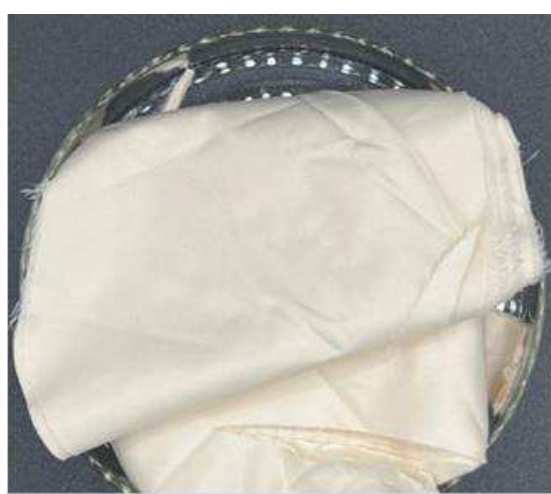

acrylic ( 20\%)

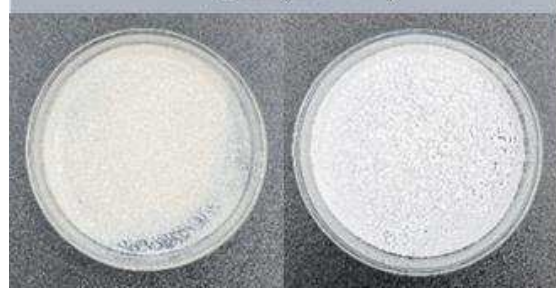

360

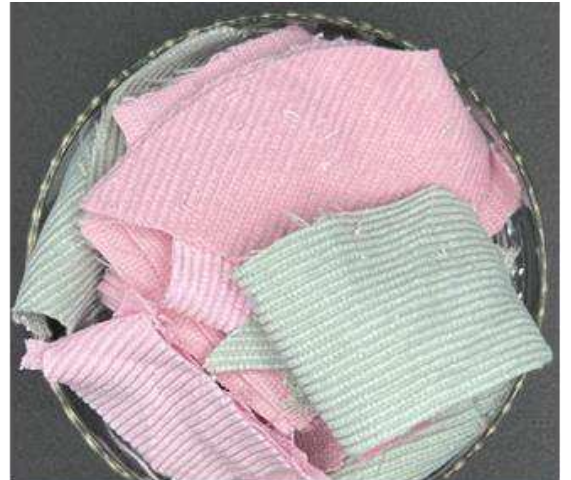

Nylon $66(\sim 5 \%)$
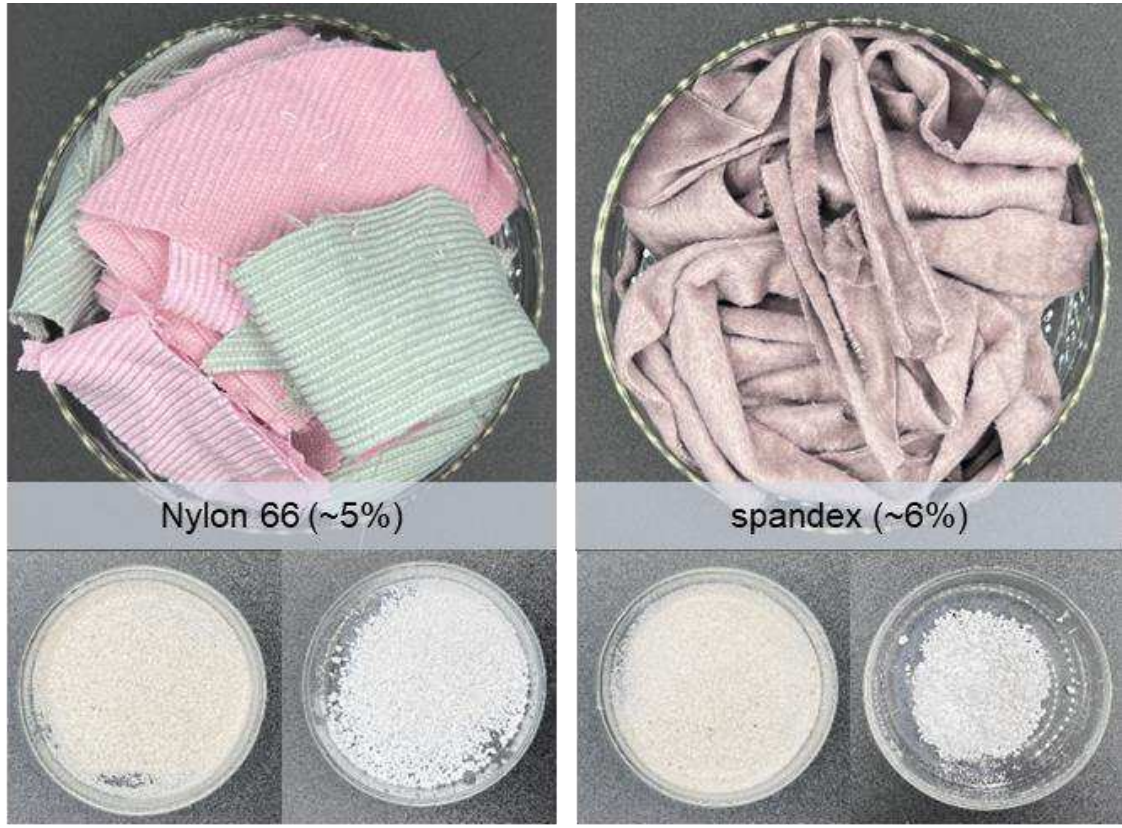

Extended Data Fig.4 Acetolysis of various textiles blended with PET. Decolorized terephthalic acid obtained from different textiles yielded greater than $90 \%$ and purity over 99.7\%. Satisfactory results of acetolysis were attributed to the excellent dissolution/decomposition effect of acetic acid on most impurities. 

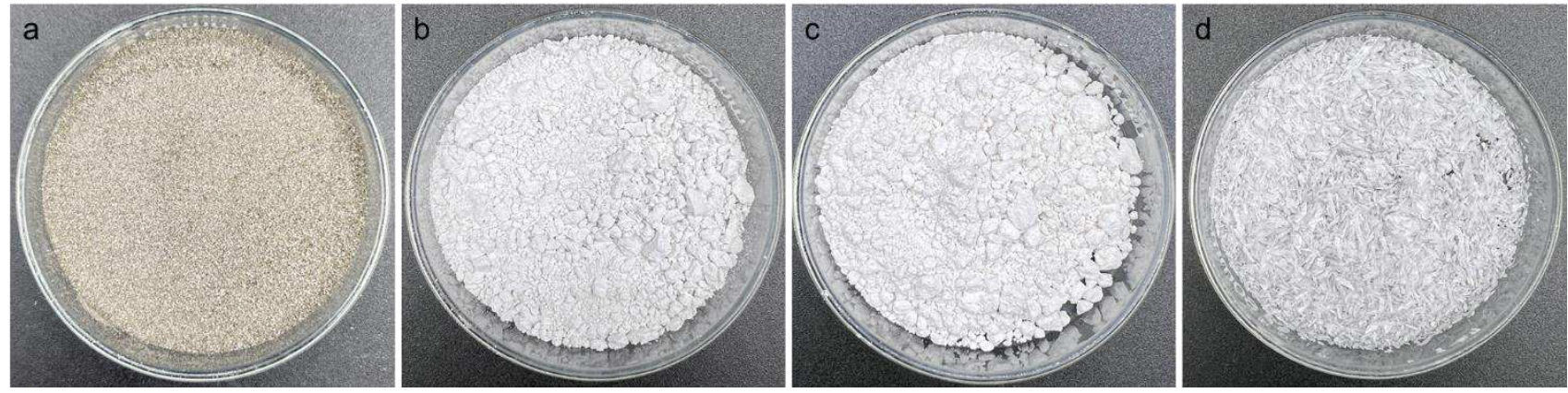

Extended Data Fig.5 Decolorization of dark TPA. a, Dark TPA obtained directly from acetolysis of Variegated clothes. b, Product obtained by decolorizing dark TPA (sample a) in an alkaline solution with activated carbon. c, Product obtained by decolorizing dark TPA (sample a) in N, N-dimethylacetamide with activated carbon. d, Product obtained by decolorizing dark TPA (sample a) in hot water $\left(280^{\circ} \mathrm{C}\right)$ with activated carbon. All dark products obtained by acetolysis of waste PET materials were easily decolorized in the described ways.

\section{Supplementary information}

372 Supplementary information includes Supplementary Notes, Tables, Figures, Video and References. 


\section{Supplementary Files}

This is a list of supplementary files associated with this preprint. Click to download.

- SI.pdf

- VideoofacetolysisofPETplastics.mp4 